\title{
Analysis of the Respiratory and Cardiac Systems Coupling in Pyramidal Exercise using a Time-Varying Model
}

\author{
O Meste $^{1}$, G Blain $^{2}$, S Bermon ${ }^{2}$ \\ ${ }^{1}$ University of Nice-Sophia Antipolis, Lab. I3S, France \\ ${ }^{2}$ University of Nice-Sophia Antipolis, Medical school, Lab. of Physiology, France
}

\begin{abstract}
The analysis of heart period series is a difficult task especially under graded exercise conditions. Among all the information present in these series, we are more interested in the coupling between respiratory and cardiac systems, known as respiratory sinus arrythmia. We show in this work that from the heart period series precise patterns concerning the respiratory frequency can be extracted. Evolutive model is introduced in order to achieve the tracking of the main frequencies and their time-varying power.
\end{abstract}

\section{Introduction}

Under resting conditions, power spectral parameters of the heart rate variability (HRV) represent mainly the autonomic nervous system influence on the sinus node. Similarly, traditional spectral analysis of the HRV during steady state maximal exercise demonstrated similarity between the high frequency band (HF) and the respiratory frequency (RF) of the studied subject. However, vaso-vagal syncope, tilt manoeuvre, acute ischemic period or graded exercise are not commonly studied due to the non-stationary behavior of the information extracted from the HRV. In the field of exercise physiology, a validated method of HRV analysis in non-stationary conditions would be of interest to study the coupling between respiratory and cardiac systems.

Prony model, usually encountered in stationary cases when damped or pure sinusoids are assumed to be recorded, makes use of an autoregressive (AR) model for the frequency estimation and a Maximum Likelihood estimation for the amplitudes of the sinusoids. In presence of time-varying frequencies, the classical way in following these variations is the tracking, where a pole representation is used. Some results have been given in [1] in case of abrupt change of the model (or the frequencies) using the RLS algorithm in order to update the values of the AR coefficients. In order to reduce the large variance of the AR coefficients estimated from the RLS algorithm we proposed to use an offline estimation of the AR coefficients introducing an evolutive Prony based model. This approach allowing a continuous variation of the AR coefficients, an effective frequency tracker is proposed based on the relation between the frequencies and the corresponding poles location. Since this first stage of the process gives only the time-varying frequencies, the remaining difficulty is to estimate the probably time-varying amplitude of the frequencies in order to define a criteria for the selection of the principal frequencies.

We will show that this approach including the Prony based evolutive model, the tracking and the time-varying filtering leads to clear results in realistic simulation and in applications where healthy subjects were administered a pyramidal exercise.

\section{Methods}

Prior to present the methodology for the processing of the HRV, we must choose the signal assumed to contain the relevant information concerning the RF. First of all we will state that the hear period (HP) signal $h p(k)=t_{k}-t_{k-1}$ (where $t_{k}$ is the occurence time of the $k$ th beat) is able to reveal the modulation signal $m(t)$ [2] containing the expected information. The first processing applied to $h p(k)$ is to remove the trend due to the varying conditions of the experiment using a polynomial fitting $p o(k)$ (order equal to 20). The second preprocessing is a 100th order highpass FIR filtering (the cut-off frequency is 0.03 with 0.5 corresponding to the half of the normalized sample rate) applied to $h p(k)$ where the trend $p o(k)$ has been removed. The resulting signal will be referenced as $m(k)$

\subsection{Frequencies estimation}

As mentioned in [3], when the signal is presenting spectral lines it can be modeled as an AR process, where the relation between the AR coefficients $a_{i}$ and the frequencies 
$f_{i}$ of the spectral lines is given by:

$$
1-\sum_{i=1}^{p} a_{i} z^{-i}=\prod_{i=1}^{p}\left(1-z_{i} z^{-1}\right), \text { where } z_{i}=e^{j 2 \pi f_{i}}
$$

Then, the frequencies $f_{i}$ are obtained computing the roots of the caracteristic polynomial given in (1). When the signal is non-stationary, the AR model doesn't stand anymore and must be replaced by:

$$
m(k)=\sum_{i=1}^{p} a_{i}(k) m(k-i)+\nu(k), \quad p+1 \leq k \leq N
$$

giving a time-varying parametric model. On the contrary to updating the AR coefficients using algorithms such RLS [1], the evolutive approach constrains the $a_{i}(k)$ to be a linear combination of some known basis functions:

$$
a_{i}(k)=\sum_{l=0}^{q} a_{i l} u_{l}(k)
$$

In this work we will use the Akaike criteria for the optimal determination of the order $q$. The order $p$ will be arbitrary set equal to 12 . The basis functions will be choosen as the orthogonal Fourier functions on the interval $[1, N-p]$. Using this linear combination, (2) can be replaced by:

$$
m(k)=\mathbf{M}(k-1) \mathbf{a}+\nu(k)
$$

where

$$
\begin{aligned}
& \mathbf{M}(k-1)=\left[u_{0}(k-1) m(k-1) u_{1}(k-1) m(k-1) \cdots\right. \\
& \left.u_{q-1}(k-1) m(k-p) u_{q}(k-1) m(k-p)\right]
\end{aligned}
$$

and

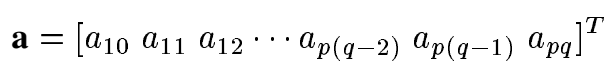

Then the parameter vector $\mathbf{a}$ is estimated using the well known least square estimator:

$$
\begin{array}{r}
\hat{\mathbf{a}}=\left[\mathbf{M}^{T} \mathbf{M}\right]^{-1} \mathbf{M}^{T} \mathbf{m} \text { where } \mathbf{M}=\left[\begin{array}{c}
\mathbf{M}(p) \\
\vdots \\
\mathbf{M}(N-1)
\end{array}\right] \\
\text { and } \mathbf{m}=[m(p+1) \ldots m(N)]^{T}
\end{array}
$$

hence $\mathbf{a}$ is estimated using (5), the $a_{i}(k)$ are computed using (3). Since in this approach we are interested in following the variations of a limited set of frequencies, the next difficulty will be to track these frequencies from the estimated timevarying $\hat{a}_{i}(k)$.

\subsection{Frequencies tracking}

Tracking varying frequencies is a difficult task where solutions correspond to given assumptions. For example, in presence of pure sinusoids efficient algorithms exist [4] but they are unfortunately not suitable with our problem since, as we will see in the application, poles deduced from (2) are not located on the unit circle. In [1], two frequency trackers are given using RLS algorithm for solving the expression (2). On the contrary to [1] the evolutive model (3) gives a continuous-time description of the $a_{i}$ allowing a more robust tracker as presented in the following.

The basic idea is to find a relation linking the derivatives of the $a_{i}(k)$ (noted $\dot{a}_{i}(k)$ ) and the corresponding $z_{i}(k)$ which are the solution of (1). This relation is obtained expanding (1) and is given by:

$$
\dot{z}_{i}(k)=\sum_{n=1}^{p} \frac{\delta z_{i}}{\delta a_{n}}(k) \dot{a}_{n}(k)
$$

where

$$
\frac{\delta z_{i}}{\delta a_{n}}(k)=\frac{z_{i}^{p-n}(k)}{\prod_{l=1 \mid l \neq i}^{p}\left(z_{i}(k)-z_{l}(k)\right)}
$$

Since an analytic formulation of $\hat{a}_{i}(k)$ (3) is available, the calculation of $\dot{a}_{n}(k)$ is straightforward. Assuming that the value obtained by (6) is equivalent to an increment, a predicted value $\tilde{z}_{i}(k+1)$ of $z_{i}(k+1)$ is given by:

$$
\tilde{z}_{i}(k+1)=z_{i}(k)+\dot{z}_{i}(k)
$$

The pole $z_{x}(k+1)$, obtained from computing the roots of (1) with the set of $a_{i}$ at index $k+1$, which minimize the distance with $\tilde{z}_{i}(k+1)$ will be chosen as a member of the poles set defining a track. Since the previous prediction can be made for each $z_{i}$, the last calculation will be to deduce the frequency tracks from the angle of the poles tracks. Obviously several problems arise due to poles variations, especially when two poles are equal or when a complex pair becomes two real poles. In order to avoid these problems due to the direct tracking on the poles, we propose to factorize (1) using order two polynomials since the order of the AR model is even, giving :

$$
\prod_{i=1}^{p}\left(1-z_{i} z^{-1}\right)=\prod_{i=1}^{p / 2}\left(1-b_{i 1} z^{-1}-b_{i 2} z^{-2}\right)
$$

Then the tracking will be achieved on the coefficients $\left(b_{i 1}, b_{i 2}\right)$ instead of $z_{i}$.

Unfortunately, the derivation, in function of time, of (9) doesn't lead to establish convenient relations of the form:

$\dot{b}_{i 1}(k)=f_{1}\left(b_{11}(k), b_{12}(k), \ldots, b_{(p / 2) 2}, \dot{a}_{1}(k), \ldots, \dot{a}_{p}(k)\right)$
$\dot{b}_{i 2}(k)=f_{1}\left(b_{11}(k), b_{12}(k), \ldots, b_{(p / 2) 2}, \dot{a}_{1}(k), \ldots, \dot{a}_{p}(k)\right)$ 
like in (6). However, in [3] we show that using a matrix formulation and omitting the index $k$ we can get the following result:

$$
\dot{\mathbf{a}}=\mathbf{B} \dot{\mathbf{b}}
$$

where

$$
\dot{\mathbf{b}}=\left[\dot{b}_{11}, \dot{b}_{12}, \ldots, \dot{b}_{(p / 2) 1}, \dot{b}_{(p / 2) 2}\right]
$$

Before to give the form of $\mathbf{B}$ we must first define the vector $\mathbf{b}_{i}$ as:

$$
\mathbf{b}_{i}=\left[\begin{array}{lll}
1 & -b_{i 1} & -b_{i 2}
\end{array}\right]^{T} .
$$

Then $\mathbf{B}$ can be defined as:

$$
\mathbf{B}=\left[\begin{array}{ccccccc}
\mathbf{c}_{1} & 0 & \mathbf{c}_{2} & 0 & \ldots & \mathbf{c}_{p / 2} & 0 \\
0 & \mathbf{c}_{1} & 0 & \mathbf{c}_{2} & & 0 & \mathbf{c}_{p / 2}
\end{array}\right]
$$

where vectors $\mathbf{c}_{i}$ are defined as:

$$
\mathbf{c}_{i}=\bigodot_{l=1 \mid l \neq i}^{p / 2} \mathbf{b}_{l}
$$

using the symbol $\odot$ as the multiple discrete convolution product.

Now adding the index $k$, the derivatives of the $b_{i 1}(k)$ 's and the $b_{i 2}(k)$ 's are obtained by:

$$
\dot{\mathbf{b}}(k)=\left(\mathbf{B}^{T}(k) \mathbf{B}(k)\right)^{-1} \mathbf{B}^{T}(k) \dot{\mathbf{a}}(k)
$$

As for the prediction of the $z_{i}(k+1)(8)$, the result from (15) will allow the prediction of the $\left(b_{i 1}(k+1), b_{i 2}(k+1)\right)$.

The global tracking procedure is summarized as following from the previously computed $a_{i}(k)$. The detailed procedure is given in [3] :

- Compute and sort the roots $z_{i}(k)$ for all $k$ of the characteristic polynomial

- Compute the $\left(b_{i 1}(k), b_{i 2}(k)\right)$ for all $i$ and $k$ using the sorted roots

- For each $k$ compute (15) in order to evaluate the predicted couple $\left(\tilde{b}_{i 1}(k+1), \tilde{b}_{i 2}(k+1)\right)=\left(b_{i 1}(k)+\dot{b}_{i 1}(k), b_{i 2}(k)+\right.$ $\left.\dot{b}_{i 2}(k)\right)$

- For each $i$ select the couple $\left(b_{i 1}(k+1), b_{i 2}(k+1)\right)$ which is the closest with the predicted one in order to append it to the $i$ th track

- Finally, each track $i$ is formed by a vector of couples corresponding to the coefficient of an order two polynomial whose roots will provide the expected tracked frequencies.

Assuming that the frequency tracks are correctly estimated, the next step is to extract the time-varying amplitudes of the spectral components described by the frequency tracks. Note that since the frequencies $f_{i}(k)$ are defined in the dimensionless interval $[-1 / 2,1 / 2]$, the multiplication of $f_{i}(k)$ by $1 / p o(k)$ gives Hertz.

\subsection{Time-varying amplitude estimation}

Several procedures are available depending on the accuracy of the previous frequency estimation. The more robust characterization is probably the calculation of the instantaneous power of the signal in frequency bands whose centers are defined by the frequency tracks. An alternative proposed in [3] is to use time-varying filters defined by the frequency tracks in order to get the phase of the signal on the contrary to only working on the power.

Defining the Short Time Fourier Transform of $m(k)$ as $\operatorname{STFT}(k, f)$ making use of an analyzing window whose length will be chosen equal to $128 \mathrm{lags}$, then the instantaneous power $I P_{i}^{\delta}(k)$ for the time-varying frequency $f_{i}(k)$ is evaluated by:

$$
I P_{i}^{\delta}(k)=\frac{1}{2} \sum_{f=f_{i}(k)-\delta}^{f_{i}(k)+\delta}|S T F T(k, f)|^{2}
$$

The value $\delta$ will be chosen depending on the window length. In the application, this value will correspond to a summation over three frequency lags.

\section{Simulation}

The aim of this simulation is to show that the global procedure is able to reveal the time-varying frequency content of the heart period signal. Instead of directly providing $h p(k)$, this simulation will produce in a first time a noisy $\left(\sigma_{\nu}=0.05\right)$ synthesized ECG $\operatorname{ecg}(n)$ which the double level technique will applied on in order to get $h p(k)$ :

$e c g(n)=\left(\cos \left(p r_{1}(n)\right)+0.03 \cos \left(p r_{2}(n)\right)+1\right)^{200}+\nu(n)$

where $p r_{1}(n)$ and $p r_{2}(n)$ will simulate a trend in the mean heart period and the modulation, respectively. The modulation $\operatorname{pr}_{2}(n)$ has been chosen in order to produce only one frequency track, as shown in fig. 1 with the result of the proposed approach. This simulation shows that the proposed evolutive modelling followed by a frequency tracker is efficient.

\section{Application}

In this application, a couple of young healthy male subjects $\left(S_{1}\right.$ and $\left.S_{2}\right)$ were administered a pyramidal exercise test on a cycle ergospimeter. After a $5 \mathrm{~min}$. warm-up session, the test consisted of a $8 \mathrm{~min}$. ramp load increase from $60 \%$ to $100 \% \dot{V} \mathrm{O} 2 \mathrm{max}$, immediately followed by a 8 min. ramp decrease from $100 \%$ to $60 \%$ $\dot{V} \mathrm{O} 2 \mathrm{max}$. Respiratory frequency (RF) was measured and stored by using an automatic ergospimeter on a breath by breath basis, and simultaneously the ECG was recorded. 


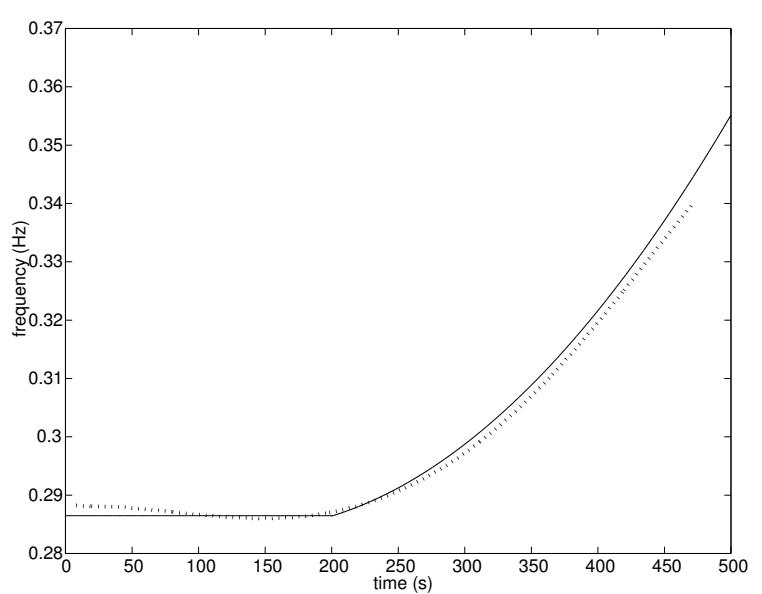

Figure 1. Time-varying frequency. The solid line and the dotted line correspond to the simulated track and the estimated track, respectively.

The previous algorithms have been applied in order to track the frequencies corresponding to the highest instantaneous power and lying in the expected frequency range. We see in fig. 2 that for both subjects the time-varying frequencies fit very well the trend of the RF. Also for both subjects, we see in fig. 3 that the increasing of the RF is accompanied by an increasing of the corresponding instantaneous power. We can note that a delay appears comparing the shape of the curves from fig. 2 and fig. 3.

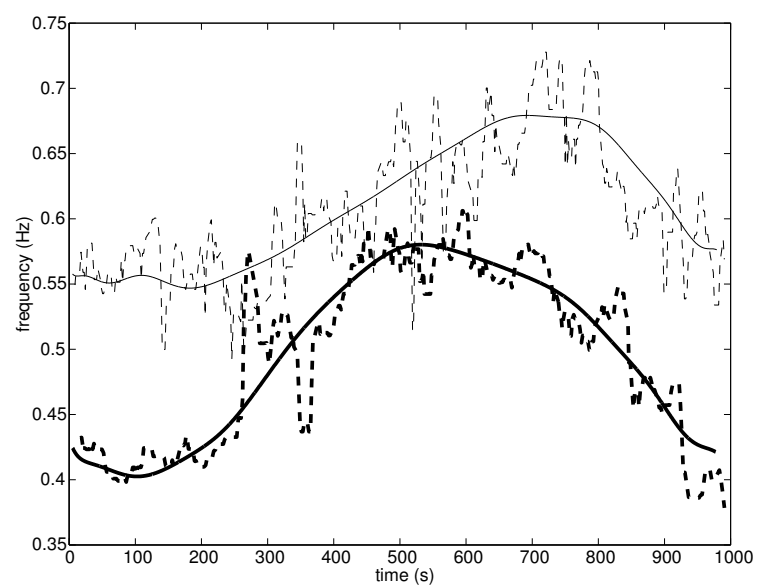

Figure 2. Respiratory frequency (dashed line) compared to the tracked frequency (solid line) for the two subjects (thin lines for $S_{1}$ and thick lines for $S_{2}$ ).

\section{Conclusion}

It is clear that the simplistic model proposed in (1) does not reflect the inherent complexity of HRV. Although investigation of HRV under exercise stress conditions tends

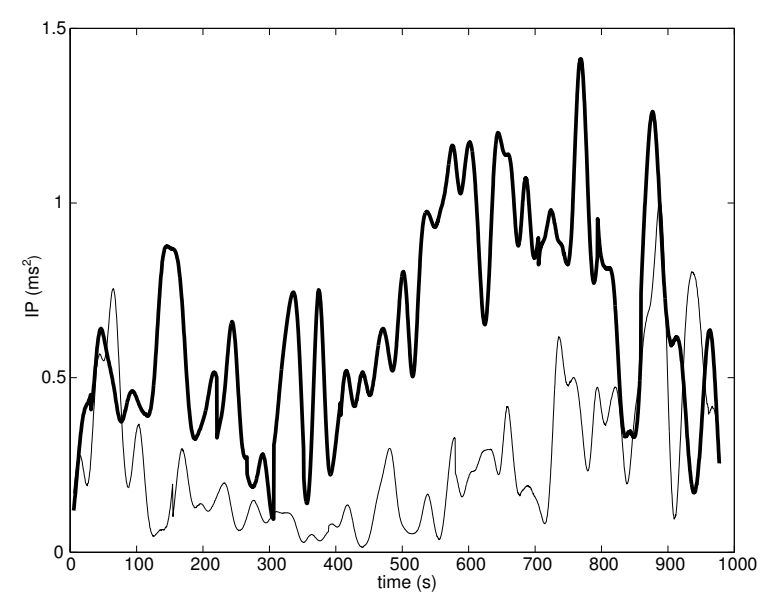

Figure 3. Instantaneous power corresponding to the tracked frequency (thin lines for $S_{1}$ and thick lines for $S_{2}$ ).

to reduce such complexity, the proposed approach is in some way robust to some modelling failure. Indeed, the presented preliminary data show that RF can be accurately predicted from the HP series analysis, and this may be relevant in the field of cardio-respiratory physiology. However, a larger set of observation is needed to state on the use of our method in the understanding of phenomenon contributing to HRV [5].

\section{References}

[1] Mainardi L, Bianchi A, Baselli G, Cerutti S. Pole-tracking algorithms for the extraction of time-variant heart rate variability spectral parameters. IEEE Trans Biomed Eng 1995;42(3):250-259.

[2] Mateo J, Laguna P. Improved heart rate variability signal analysis from the beat occurence times according to the ipfm model. IEEE Trans Biomed Eng 2000;47(8):985-996.

[3] Meste O, Blain G, Bermons S. Analysis of non-stationary signal using frequency tracking and time-variant filtering. I3S/RR 2002-39-FR, Lab I3S, 2002.

[4] Strobach P. The recursive companion matrix root tracker. IEEE Trans Signal Processing 1997;45(8):1931-1942.

[5] Cammann H, Michel J. How to avoid misinterpretation of heart rate variability power spectra ? Computer Methods and Programs in Biomedicine 2002;68:15-23.

Address for correspondence:

Olivier Meste

Lab. I3S, UNSA-CNRS

2000, route de Lucioles. BP 121

06903 Sophia Antipolis Cedex, France

tel./fax: +330492965104 / +330492965155

meste@i3s.unice.fr 